\title{
Intelligent Regulation Algorithm of Automobile Rear View Mirror based on Eye Location
}

\author{
Lihua $\mathrm{Wu}^{\mathrm{a},{ }^{*},}$ Xu Bai ${ }^{\mathrm{a}}$, Dianshuang Zheng ${ }^{\mathrm{b}}$, and Jianxin Gai ${ }^{\mathrm{a}}$ \\ ${ }^{a}$ School of Measurement Control Technology and Communications Engineering, Harbin University of Science and Technology, Harbin, 150080, China \\ ${ }^{b}$ Harbin Jiancheng Group Co., Ltd., Harbin, 150080, China
}

\begin{abstract}
For the defects in the automobile rear view mirror regulation method, an intelligent regulation algorithm of automobile rear view mirrors is studied in this paper. The algorithm utilizes drivers' images in vehicles gathered by a single camera to determine the practical spatial positions of drivers' binocular centers in the vehicles. Then, through the regulation relationship algorithm between eye location and the rear view mirror, the intelligent regulation of optimal view of automobile rear view mirrors is achieved. The experimental results indicate that the algorithm has the advantages of fast regulation, convenience, and accuracy.
\end{abstract}

Keywords: automobile rear view mirror; intelligent regulation; correction of distorted coordinate point; optimal view

(Submitted on July 16, 2019; Revised on August 15, 2019; Accepted on September 23, 2019)

(C) 2019 Totem Publisher, Inc. All rights reserved.

\section{Introduction}

With the rapid development of the automobile industry in recent years, the quantity of cars in China has increased. Meanwhile, casualties and property losses caused by traffic accidents have also increased each year [1]. The survey shows that some accidents happen because drivers fail to observe their surroundings sufficiently. As an important component of automobiles, the rear view mirror plays a significant role in safe driving and parking [2]. At present, regulation methods of rear view mirrors mainly include manual regulation and electric regulation. When drivers are different in stature, they need to regulate and change the inclination angle of the rear view mirror based on their perspective [3]. The optimal view scope of the automobile left rear view mirror is shown in Figure 1. By regulating the inclination angle of rear view mirrors, the distant horizon line can be located at the vertical center location of the image in the rear view mirror. The area of the vehicle body edge accounts for $1 / 4$ of the image in the rear view mirror [4]. Most new drivers and even some experienced drivers fail to master the optimal view angle of rear view mirrors. Sometimes, drivers regulate rear view mirrors during the driving process, which poses dangers. Studying the intelligent regulation algorithm of rear view mirrors carries important practical and theoretical significance.

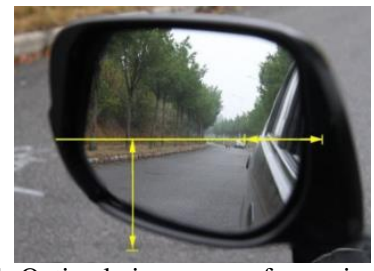

Figure 1. Optimal view scope of rear view mirrors

\section{Calculation of Practical Location of Eye Center}

To confirm the optimal view of rear view mirrors for different drivers, it is necessary to obtain the driver's location as the

* Corresponding author.

E-mail address: 13624609698@126.com 
actual space coordinates in the cab. The side view diagram of a driver in the vehicle is shown in Figure 2. The actual space coordinate system of the cab is set as $\mathrm{O}-\mathrm{XYZ}$, and the $Y$ axis is perpendicular to the automotive chassis.

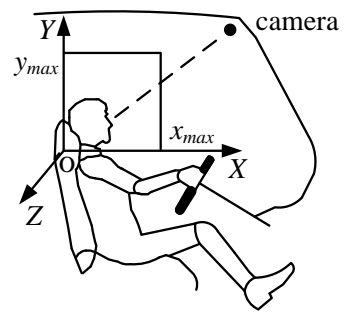

Figure 2. Side view of driver in cab

Due to the structure of the cab, the camera is installed exactly above the driver in order to not block the driver's view. While installing the camera, images with known size are shot to calibrate the camera so as to confirm the location of the original point of image coordinates in the actual coordinate system [5].

The driver is in the 3D space $O-X Y Z$ in the vehicle. In normal conditions, the driver does not need to move their body to the left and right in the driving process. In other words, the driver will not move in the $Z$ axis direction, that is, the practical coordinate of eyes on the $Z$ axis is $z=0$. Thus, the eye center location of different drivers only changes on the $X$ axis and $Y$ axis.

In Figure 2, the area that consists of coordinate points $\left(x_{\max }, 0\right)$ and $\left(0, y_{\max }\right)$ is the change scope of binocular center locations of drivers with different stature. When different drivers drive normally, changes in the binocular center location will not exceed this scope. According to different vehicle models, $x_{\max }$ and $y_{\max }$ are less than $60 \mathrm{~cm}$ in normal conditions.

\subsection{Calculation of Eye Center Coordinates in Perspective Image}

Firstly, the algorithm needs to carry out eye positioning for the collected images of the driver. The binoculars nearest to the $Y$ axis and to the original point are chosen as the driver's eye coordinates.

The OpenCV-based Adaboost cascade classifier algorithm is applied in this paper. The target detection classifier in the "haarcascad_eye.xml" file is used to transform the images [6-7]. Since the cascade classifier selects haar features, the perspective image needs to be transformed into grayscale images in the eye positioning calculation, and 2/5 parts of the human face area are searched to find the eye area [8-9]. The coordinates $\left(u_{l}, v_{l}\right)$ and $\left(u_{r}, v_{r}\right)$ of the two eyes in the image coordinate system $\mathrm{O}^{\prime}-U V$ are gained, as shown in Figure 3. The binocular coordinates are averaged to obtain the binocular center coordinates.

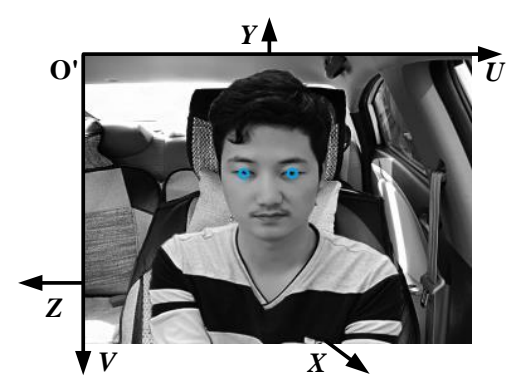

Figure 3. Eye positioning map

In Figure 3, to gain eye locations in images and the actual space coordinate system, it is necessary to determine the original point $\mathrm{O}^{\prime}$ of the image coordinate system in the $Y Z$ plane of the actual space coordinate system. The coordinate of $\mathrm{O}^{\prime}$ in the actual space coordinate system is $(d, w / 2) . d$ is the location of image size in the $Y$ axis direction in corresponding actual space. $w$ is the location of image size in the $Z$ axis direction in the corresponding actual space. The unit of the actual space coordinate system is set as centimeters, and the unit of the image coordinate system is pixels. To improve the computing speed, the image pixel involved is set as $U_{\max } \times V_{\max }=640 \times 480$ under the precondition of ensuring the precision. Through calibration, the image size in the corresponding actual space is $w \times d=75 \mathrm{~cm} \times 55 \mathrm{~cm}$, that is, $1 \mathrm{~cm}$ in the horizontal direction corresponds to 8.53 pixels, and $1 \mathrm{~cm}$ in the vertical direction corresponds to 8.72 pixels. Thus, the original point $\mathrm{O}^{\prime}$ of the image coordinate system in the actual space coordinate system is (55, 37.5). The binocular 
coordinates gained by eye positioning are $(265,198)$ and $(365,196)$. After averaging, the binocular center coordinates are $(u$ $=315, v=197$ ).

\subsection{Correction Algorithm of Distorted Coordinate Point}

Since the camera is installed exactly above the driver, the collected image is not the driver's front view but the perspective image with certain nonlinear distortion. Thus, it is necessary to carry out distortion correction for the perspective image so as to obtain the actual coordinate values $(y, z)$ of the driver's binocular center [10-12]. Normally, $z$ approximates 0 . The general approach is to carry out distortion correction for the whole image, but the calculation quantity is large [13]. In this paper, only the eye location in the perspective image is corrected, because only the driver's eye location will influence the regulation angle of rear view mirrors. The correction algorithm of the distorted coordinate point selects multiple fixed known points in the cab front views, finds the corresponding points in the cab perspective images, and substitutes these points into the space coordinate transformation formula to determine the correction equation coefficient of the distorted coordinate point, thus gaining the correction equation of the distorted coordinate point. The binocular center coordinates $(u$, $v$ ) in the perspective image is substituted into the correction equation of the distorted coordinate point to obtain the corrected image coordinates $\left(u^{\prime}, v^{\prime}\right)$.

The corrected image coordinates $\left(u^{\prime}, v^{\prime}\right)$ and the coordinates in the perspective image have the relationship shown in Equation (1) [14].

$$
\left\{\begin{array}{l}
u^{\prime}=h_{1}(u, v) \\
v^{\prime}=h_{2}(u, v)
\end{array}\right.
$$

Where $h_{1}(u, v)$ and $h_{2}(u, v)$ are nonlinear functions that can be approximated by the space coordinate transformation formula [15], as shown in Equation (2).

$$
\left\{\begin{array}{l}
u^{\prime}=\sum_{i=0}^{n} \sum_{j=0}^{n-i} b_{i j} u^{j} v^{i} \\
v^{\prime}=\sum_{i=0}^{n} \sum_{j=0}^{n-i} a_{i j} u^{j} v^{i}
\end{array}\right.
$$

In Equation (2), $n$ is the order of the polynomial, and $a_{i j}$ and $b_{i j}$ are undetermined coefficients. As long as the undetermined coefficients are confirmed, the relationship between the distorted image coordinate and corrected image coordinate can be determined. As previously mentioned, the distortion of coordinate points in the perspective image is nonlinear. The larger the value of $n$, the smaller the error. However, the computing time will be longer. Within the allowable scope of correction error of the distorted coordinate point, $n=2$ is taken in Equation (2), and the corrected nonlinear transformation equation of the distorted coordinate point is gained, as shown in Equation (3).

$$
\left\{\begin{array}{l}
u^{\prime}=b_{00}+b_{10} v+b_{01} u+b_{20} v^{2}+b_{11} v u+b_{02} u^{2} \\
v^{\prime}=a_{00}+a_{10} v+a_{01} u+a_{20} v^{2}+a_{11} v u+a_{02} u^{2}
\end{array}\right.
$$

In order to make the correction result of distorted coordinate point more accurate, least squares fit is used to calculate the coefficients $a_{i j}$ and $b_{i j}$. Since it is only necessary to correct the coordinate value of the driver's binocular center in the perspective on the $V$ axis, only $v^{\prime}$ in Equation (3) needs to be fitted.

In the correction algorithm of the distorted coordinate point, one front view is shot in the horizontal direction of the cab (Figure 4), and one perspective image is shot by the camera (Figure 5).

In view of the influence of regulation precision, algorithm operation speed, and passengers on sheltering known points, ten fixed known points $\left(u_{i}^{\prime}, v_{i}^{\prime}\right)(i=1,2,3, \cdots, 10)$ marked with " $x^{\prime \prime}$ are shown in Figure 4 . These points have the corresponding points $\left(u_{j}, v_{j}\right)$ with the same features in the perspective image of Figure 5 , and $j=1,2,3, \cdots, 10$. The image coordinate table of known points is shown in Table 1. 

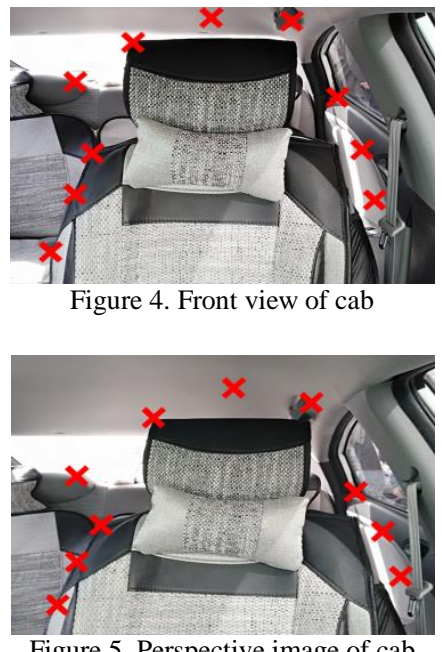

Table 1. Coordinate table of known points (unit: pixel)

\begin{tabular}{|c|c|c|}
\hline No. & Perspective image coordinate & Front view coordinates \\
\hline 1 & $(297,34)$ & $(320,56)$ \\
\hline 2 & $(426,58)$ & $(210,109)$ \\
\hline 3 & $(185,59)$ & $(149,226)$ \\
\hline 4 & $(137,145)$ & $(510,235)$ \\
\hline 5 & $(492,155)$ & $(129,291)$ \\
\hline 6 & $(129,210)$ & $(526,299)$ \\
\hline 7 & $(497,247)$ & $(72,338)$ \\
\hline 9 & $(80,253)$ & $(542,391)$ \\
\hline 10 & $(514,331)$ & $(70,427)$ \\
\hline
\end{tabular}

The coordinate points in Table 1 are used, and least squares fit is applied to determine the value of coefficient $a_{i j}$. Under the condition of guaranteeing system accuracy, four decimals are retained for the calculation result. The values of $a_{00}$, $a_{10}, a_{01}, a_{20}, a_{11}$, and $a_{02}$ are $142.9956,-0.7861,1.2469,0.0012,0.0001$, and -0.0009 , respectively.

After the values of $a_{00}, a_{10}, a_{01}, a_{20}, a_{11}$, and $a_{02}$ are substituted into Equation (3), the correction equation of the coordinate points of the driver's binocular center on the $V$ axis can be gained, as shown in Equation (4).

$$
v^{\prime}=142.9956-0.7861 v+1.2469 u+0.0012 v^{2}+0.0001 v u-0.0009 u^{2}
$$

The binocular center coordinates $(u=315, v=197)$ in the perspective image is substituted into Equation (4) to get the coordinate $v^{\prime}$ of the corrected binocular center on the $V$ axis. The transformational relation of the $Y$ axis coordinate $y$ and the $V$ axis coordinate $v^{\prime}$ of the driver's binocular center is shown in Equation (5).

$$
y=d-\frac{d \times v^{\prime}}{V_{\max }}
$$

In Equation (5), $d$ has been calibrated in Section 1.1. $V_{\max }$ is the maximum pixel in the vertical direction, namely, 480. $v^{\prime}$ is substituted into Equation (5) to gain the actual coordinate $y$ on the $Y$ axis of the driver's binocular center.

\subsection{Calculation of Actual Location of Eye Center on X Axis}

Based on the above calculation, only the actual location of the driver's binocular center on the $Y$ axis can be obtained. To determine the actual location of eyes on the $X$ axis, the monocular vision distance measurement is required to gain the actual coordinates of the driver's binocular center on the $X$ axis. The model of monocular vision distance measurement is shown in Figure 6 [16-17].

In Figure 6, $\mathrm{O}^{\prime}-U V$ is the image coordinate system, and $\mathrm{O}-X Y Z$ is the actual space coordinate system. Point $N$ is the location of the camera, and the height from the camera to the $X$ axis is $N M=H$. The projection point of the binocular center 
coordinate point $P$ on the image coordinate system is $p^{\prime}$. The coordinates of $p^{\prime}$ are $\left(u^{\prime}, v^{\prime}\right)$. Point $e$ is the central point of the image, and it corresponds to point $E$ in the actual coordinate system. The length of $E M$ can be gained by camera calibration. $\alpha$ is the included angle of the optical axis and the $X$ axis. The included angle of $P N$ and the optical axis is $\gamma . Q M$ is the distance from the driver's binocular center to the $X$ axis, and $Q M=h=y$. OM is the distance from the camera to the $Y$ axis. The installation location and angle of the camera decide the values of $\alpha, \gamma, H, E M$, and $O M$. The focal length of the camera is $e N=f$, where the value of $f$ is decided by camera parameters.

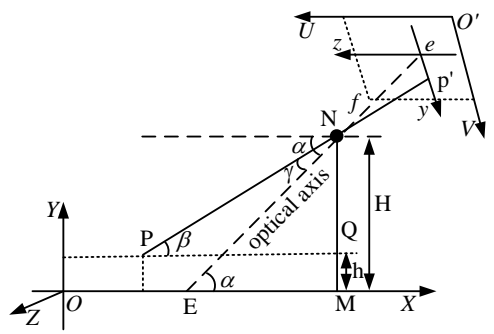

Figure 6. Algorithm model of monocular vision distance measurement

The following relations are gained by the known conditions in Figure 6.

$$
\begin{gathered}
\alpha=\arctan \left(\frac{H}{E M}\right) \\
\gamma=\arctan \left(\frac{e p^{\prime}}{e N}\right)=\arctan \left(\frac{v^{\prime}}{f}\right) \\
P Q=\frac{N M-Q M}{\tan \beta}=\frac{H-y}{\tan \left[\alpha-\arctan \left(\frac{v^{\prime}}{f}\right)\right]} \\
x=O M-P Q
\end{gathered}
$$

The distance $P Q$ from the driver's binocular center to the camera can be gained from Equation (8), and it is substituted into Equation (9) to get the actual coordinate $x$ on the $X$ axis of the driver's binocular center.

Based on the above computational algorithm, the actual space coordinates $(x, y)$ of the driver's binocular center in the vehicle can be obtained.

\section{Algorithm of Rear View Mirror Regulation Angle}

In this paper, regulating the optimal view of the left rear view mirror is taken as an example to deduce the relationship between the actual coordinates of the binocular center and the rear view mirror regulation angle.

\subsection{Relational Algorithm Between Actual Coordinate of Binocular Center and Rear View Mirror Regulation Angle}

The rear view mirror rotation diagram in the horizontal direction is shown in Figure 7. When the binocular center location of different drivers changes on the $X$ axis, the rear view mirror needs to be rotated around the $I$ axis in the horizontal direction. The activity range of the rear view mirror in the horizontal direction can be regulated to the maximum $\alpha_{\max }$ from 0 .

When the driver's binocular center is at $x=0$, the rotation angle of the rear view mirror around the $I$ axis in the horizontal direction to the optimal location is set as 0 . When the driver's binocular center is at $x_{\max }$, the rear view mirror is rotated around the $I$ axis in the horizontal direction to $\alpha_{\max }$. Thus, the regulation parameter $a$ of the rear view mirror in the horizontal direction is gained, as shown in Equation (10).

$$
a=\alpha_{\max } / x_{\max }
$$


The rotation diagram of the rear view mirror in the vertical direction is shown in Figure 8 . When the binocular center of different drivers changes on the $Y$ axis, the rear view mirror needs to be rotated around the $J$ axis in the vertical direction. The activity range of the rear view mirror in the vertical direction can be regulated to the maximum $\beta_{\max }$ from 0 .

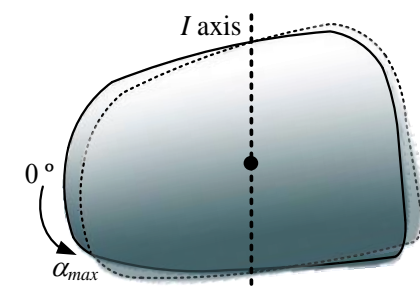

Figure 7. Rear view mirror rotation diagram at horizontal direction

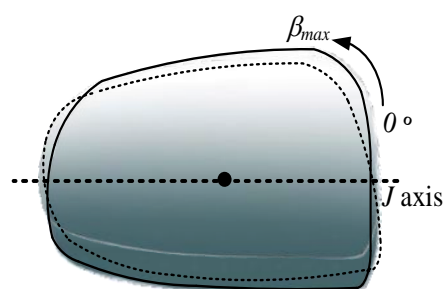

Figure 8. Rear view mirror rotation diagram at vertical direction

When the driver's binocular center is at $y=0$, the rotation angle of the rear view mirror around the $J$ axis in the vertical direction to the optimal location is set as 0 . When the driver's binocular center is at $y_{\max }$, the rear view mirror is rotated around the $J$ axis in the vertical direction to $\beta_{\max }$. Thus, the regulation parameter $b$ of the rear view mirror in the vertical direction is gained, as shown in Equation (11).

$$
b=\beta_{\max } / y_{\max }
$$

Through Equations (10) and (11), the algorithm can gain the regulation angle and angle increment of rear view mirrors in the horizontal direction and vertical direction, as below:

$$
\begin{gathered}
\left\{\begin{array}{l}
\alpha_{k}=a x \\
\beta_{k}=b y
\end{array}\right. \\
\left\{\begin{array}{l}
\Delta \alpha=\alpha_{k}-\alpha_{k-1} \\
\Delta \beta=\beta_{k}-\beta_{k-1}
\end{array}\right.
\end{gathered}
$$

In Equation (12), the values of parameters $a$ and $b$ can be decided by cab structure and mechanical transformation structure of the rear view mirror. In Equation (13), $\alpha_{k}$ and $\beta_{k}$ are the original angles of rear view mirrors, and $\alpha_{k-1}$ and $\beta_{k-1}$ are the angles to be regulated.

Because the distance and angle of drivers relative to the left and right rear view mirrors differ, the regulation relation of the actual coordinates of the binocular center with left and right rear view mirrors also differs. In the regulation formulas of left and right rear view mirrors, only the values of the coefficients $a$ and $b$ differ.

\subsection{Computational Algorithm of Pulse Value of Stepping Motor}

In order to allow the driver to gain the optimal view range from the rear view mirror as shown in Figure 1, it is necessary to regulate rear view mirrors according to different locations of the driver in the vehicle to make the rear view mirrors form a certain angle with the driver's binocular center. The inclination angle of rear view mirrors is regulated through the stepping motor, which controls the mechanical structure of worm and gear [18-19]. Hence, the algorithm needs to calculate the pulse number input to the stepping motor to regulate the rotation angle of rear view mirrors [20-21]. It is supposed that when the rear view mirror rotates in the horizontal direction (as shown in Figure 7), $n$ pulses are input into the stepping motor, which controls the rotation of rear view mirrors in the horizontal direction. Then, the rear view mirror will rotate one degree around the $I$ axis in the horizontal direction. When the rear view mirror rotates in the vertical direction (as shown in Figure 
8), $m$ pulses are input into the stepping motor, which controls the rotation of rear view mirrors in the vertical direction. Then, the rear view mirror will rotate one degree around the $J$ axis in the vertical direction. $n$ and $m$ are the conversion ratios of the rotation angle of the rear view mirror and the rotation angle of the stepping motor, respectively. The mechanical transformation structure in the rear view mirror is different, and the values of $n$ and $m$ also differ. The computational formula of the input pulse number of the stepping motor is shown as Equation (14).

$$
\left\{\begin{array}{l}
T=n \times \Delta \alpha \\
L=m \times \Delta \beta
\end{array}\right.
$$

In Equation (14), $T$ represents the pulse number input to the corresponding motor when the rear view mirror is regulated in the horizontal direction, and $L$ refers to the pulse number input to the corresponding motor when the rear view mirror is regulated in the vertical direction. The forward or backward rotation of the corresponding motor is decided by the symbol $\Delta \alpha$ or $\Delta \beta$ in Equation (14). When $\Delta \alpha$ or $\Delta \beta$ is positive, the stepping motor rotates forward. When $\Delta \alpha$ or $\Delta \beta$ is negative, the stepping motor rotates backward.

\section{Test Results}

To test the performance of the proposed algorithm, the camera is installed at the site shown in Figure 2. The distance from the camera to the $X$ axis is $54 \mathrm{~cm}$, and the included angle between the optical axis of the camera and the $X$ axis is $30^{\circ}$. Based on the above conditions, ten perspective images of drivers with different stature are shot, as shown in Figure 9 . To calculate the algorithm error, the front views of the above drivers are shot, as shown in Figure 10.

By comparing binocular center coordinates in the front views and binocular center coordinates in the actual binocular center, it is found that although an error exists in the two, the error is small and can be neglected. Therefore, binocular coordinates in the front views can replace the actual binocular coordinates.

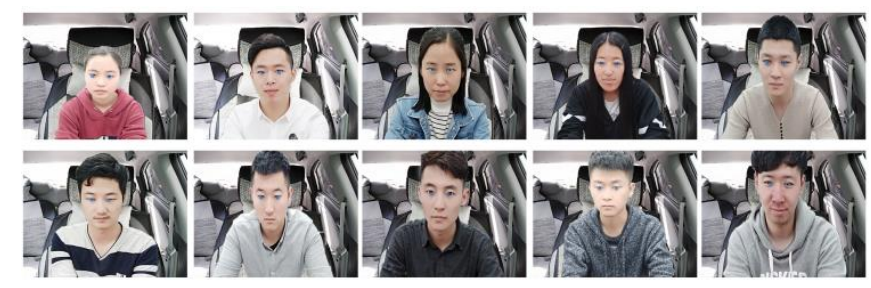

Figure 9. Perspective images of different drivers

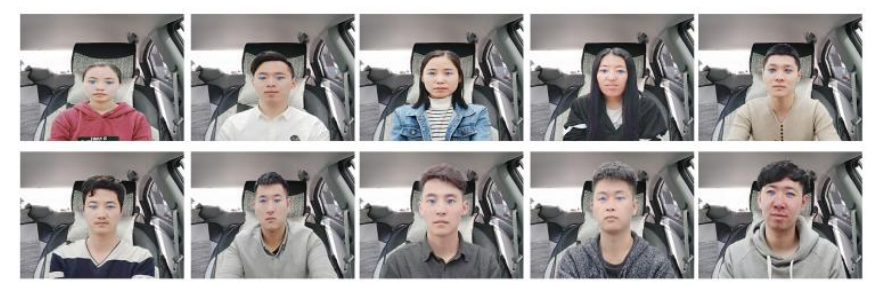

Figure 10. Front views of different drivers

Eye positioning is conducted for ten perspective images of drivers in Figure 9, and the coordinate curve of eye images and the calculation curve of the eye positioning algorithm are drawn, as shown in Figure 11. Correction of distorted coordinate points is conducted for ten groups of binocular center coordinates gained from eye positioning to obtain the actual location of the drivers on the $Y$ axis. The actual location of the drivers' binocular centers on the $X$ axis is obtained by monocular vision distance measurement. The curve of the actual eye location is shown in Figure 12.

The solid line in Figure 11 represents the image coordinates of the binocular center location gained from the perspective image by the mouse on the computer screen, and the dotted line refers to the image coordinates of the binocular center calculated from eye positioning. It can be seen from the figure that the maximum error of the eye positioning algorithm at the horizontal level is 10 pixels, and the average error is 6.9 pixels. The maximum error in the vertical direction is 8 pixels, and the average error is 5.2 pixels. The solid line in Figure 12 represents the measured actual coordinates of the binocular center, and the dotted line refers to the actual coordinates of the binocular center gained by the distorted coordinate point correction algorithm and the monocular vision distance measurement algorithm. According to Figure 12, the maximum 
error of the distorted coordinate point correction algorithm is $1.1 \mathrm{~cm}$, and the average error is $0.68 \mathrm{~cm}$. The maximum error of the monocular vision distance measurement algorithm is $1.18 \mathrm{~cm}$, and the average error is $0.79 \mathrm{~cm}$.

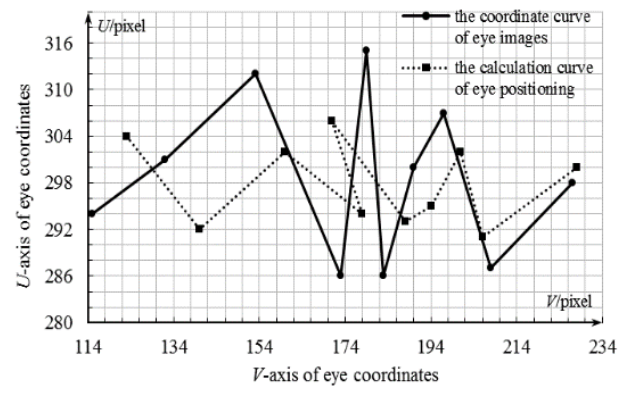

Figure 11. Eye positioning curve

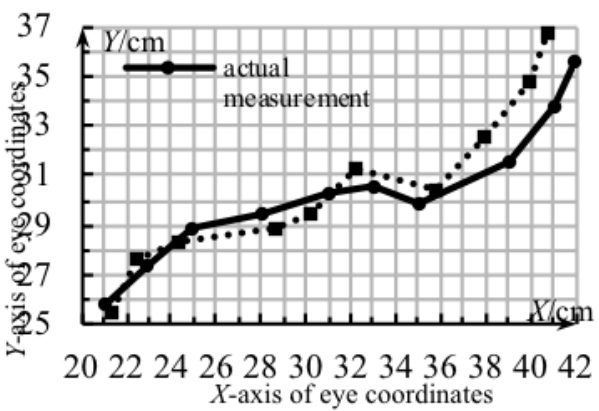

Figure 12. Actual eye coordinate curve

The conversion ratios of the rotation angle of the stepping motor and the rotation angle of the rear view mirror are respectively selected as $n=106.4$ and $m=138.8$. The values of $\alpha_{\max }, \beta_{\max }, x_{\max }$, and $y_{\max }$ are measured by the algorithm. The experimental parameters are $a=0.73$ and $b=0.64$. According to the ten groups of the actual coordinates of the driver's binocular center, the curve of rear view mirror angle regulation can be gained, as shown in Figure 13.

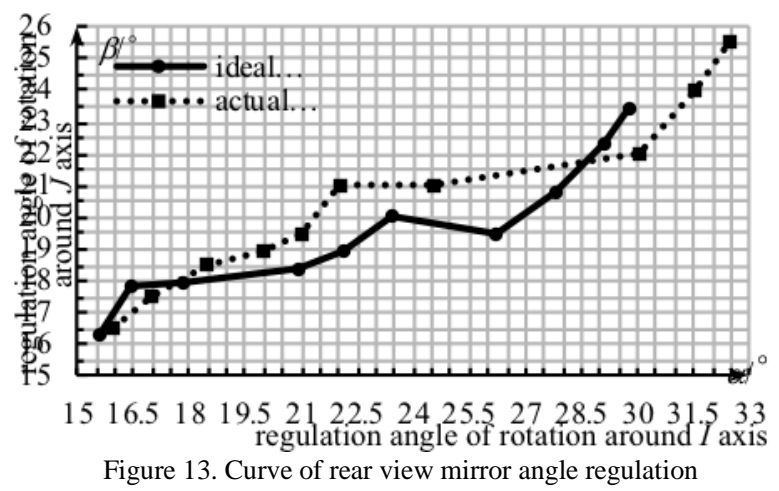

In Figure 13, the solid line represents the ideal regulation angle of the rear view mirror, and the dotted line refers to the actual regulation angle of the rear view mirror. It can be seen from the figure that the maximum error of rear view mirror rotation around the $I$ axis is $2.7^{\circ}$, and the average error is $1.41^{\circ}$. The maximum error of rear view mirror rotation around the $J$ axis is $2^{\circ}$, and the average error is $0.96^{\circ}$.

\section{Conclusions}

The intelligent regulation algorithm of automobile rear view mirrors retains the original functions of the rear view mirror regulating system. The algorithm achieves intelligent rear view mirror regulation and also provides a safety guarantee for drivers who are unfamiliar with optimal rear view mirror view regulation. When the algorithm is used in vehicle models with different specifications, it is only necessary to modify relevant regulation parameters. 
Acknowledgements

This work was supported by the National Natural Science Foundation of China (NSFC) (No. 61501150).

\section{References}

1. Y. Zhang, "The Socioeconomic Characteristics, Urban Built Environment and Household Car Ownership in a Rapidly Growing City: Evidence from Zhongshan," Journal of Asian Architecture and Building Engineering, Vol. 17, No. 1, pp. 133-140, January 2018

2. G. Knaap, U. Avin, and L. Fang, "Driving and Compact Growth: A Careful Look in the Rearview Mirror," Journal of the American Planning Association, Vol. 83, No. 1, pp. 32-35, December 2017

3. C. Ben and C. Kiron, "Changes in Level of Household Car Ownership: The Role of Life Events and Spatial Context," Transportation, Vol. 43, No. 4, pp. 565-599, July 2016

4. L. Blum, "An Unexpected Sight in the Rearview Mirror," American Journal of Psychiatry, Vol. 174, No. 9, pp. 832-834, September 2017

5. O. Korkalo and T. Tikkanen, "Auto-Calibration of Depth Camera Networks for People Tracking," Machine Vision and Applications, Vol. 30, No. 4, pp. 671-688, June 2019

6. D. F. Jin and Y. Yang, "Using Distortion Correction to Improve the Precision of Camera Calibration," Optical Review, Vol. 26, No. 2, pp. 269-277, April 2019

7. S. G. Konov and B. N. Markov, "Algorithm of Correction of Error Caused by Perspective Distortions of Measuring Mark Images," Mechanics and Industry, Vol. 17, No. 7, pp. 713-719, July 2016

8. I. Abdelhameed and T. Alaa, "Optimized Superpixel and AdaBoost Classifier for Human Thermal Face Recognition," Signal Image and Video Processing, Vol. 4, No. 12, pp. 711-719, May 2018

9. Z. Y. Zhang, X. H. Guo, and Y. Lin, "Trust Management Method of D2D Communication based on RF Fingerprint Identification," IEEE Access, No. 6, pp. 66082-66087, October 2018

10. M. Wang and W. Y. Chen, "Blink Detection using Adaboost and Contour Circle for Fatigue Recognition," Computers and Electrical Engineering, Vol. 58, No. 9, pp. 487-492, February 2017

11. Y. Lin, X. Zhu, Z. Zheng, Z. Dou, and R. Zhou, "The Individual Identification Method of Wireless Device based on Dimensionality Reduction and Machine Learning," Journal of Supercomputing, Vol. 75, No. 5, pp. 1-18, June 2017

12. Y. Lin, C. Wang, J. X. Wang, and Z. Dou, “A Novel Dynamic Spectrum Access Framework based on Reinforcement Learning for Cognitive Radio Sensor Networks," Sensors, Vol. 16, No. 10, pp. 1-22, October 2016

13. X. H. Wu and R. J. Hu, "Pedestrian Traffic Light Detection in Complex Scene using AdaBoost with Multi-Layer Features," Journal of Engineering Research, Vol .6, No. 3, pp. 34-53, September 2018

14. F. Q. Zhou, X. Chen, and H. S. Tan, "Double Distortion Correction Method in a Catadioptric Vision System with a Conic Mirror," Journal of the Optical Society of America a-Optics Image Science and Vision, Vol. 34, No. 9, pp. 1651-1658, September 2017

15. M. Sljivaric, M. Rezo, and L. Grgic, "Methods of Modelling the Distortion Caused by Different Amount and Orientation of Coordinate Corrections Between Two Coordinate Systems at Various Locations," Tehnicki Vjesnik Technical Gazette, Vol. 25, No. 1, pp. 1-9, February 2018

16. J. W. Yu, "Design and Experiment of Distance Measuring System with Single Camera for Picking Robot," Transducer and Microsystem Technologies, Vol. 3, No. 15, pp. 229-233, June 2010

17. L. F. Luo, "Design of Virtual Test System based on Hardware-in-Loop for Picking Robot Vision Localization and Behavior Control," Transactions of the Chinese Society of Agricultural Engineering, Vol. 4, No. 33, pp. 39-46, February 2017

18. K. Borell and R. Johansson, "In the Rearview Mirror," Sociologisk Forskning, Vol. 50, No. 1, pp. 3-5, January 2013

19. M. F. Cataldo, "Driving with the Rear View Mirror," Behavior Analyst, Vol. 40, No. 1, pp. 161-165, June 2017

20. Y. Tu, Y. Lin, J. Wang, J. -U. Kim, "Semi-Supervised Learning with Generative Adversarial Networks on Digital Signal Modulation Classification," CMC-Computers Materials and Continua, Vol. 55, No. 2, pp. 243-254, May 2018

21. Y. Lin, Y. Li, X. Yin, and Z. Dou, "Multisensor Fault Diagnosis Modeling based on the Evidence Theory," IEEE Transactions on Reliability, Vol. 67, No. 2, pp. 513-521, August 2018

Lihua Wu is a professor in the School of Measurement and Communication at Harbin University of Science and Technology. Her research mainly focuses on signal and information processing.

Xu Bai is a master's student in the School of Measurement and Communication at Harbin University of Science and Technology. His major is instrument science and technology.

Dianshuang Zheng is a senior engineer at Harbin Jiancheng Group Co., Ltd. His major is instrument science and technology.

Jianxin Gai is an associate professor in the School of Measurement and Communication at Harbin University of Science and Technology. His research mainly focuses on signal and information processing. 